\title{
Development of full-field deflectometry for characterization of free-form mirrors for space applications
}

Jean-François Vandenrijt, Arno Bouwens, Luc Boussemaere, Philippe Antoine, Vincent Moreau, et al.

Jean-François Vandenriit, Arno Bouwens, Luc Boussemaere, Philippe Antoine, Vincent Moreau, Benoït Borguet, Mathijs Arts, Dana Tomuta, Marc Georges, Jean-François Vandenrijt, "Development of full-field deflectometry for characterization of free-form mirrors for space applications," Proc. SPIE 11852, International Conference on Space Optics - ICSO 2020, 118526I (11 June 2021); doi: 10.1117/12.2600299

SPIE Event: International Conference on Space Optics - ICSO 2021, 2021, Online Only 


\section{International Conference on Space Optics-ICSO 2020}

Virtual Conference

30 March-2 April 2021

Edited by Bruno Cugny, Zoran Sodnik, and Nikos Karafolas
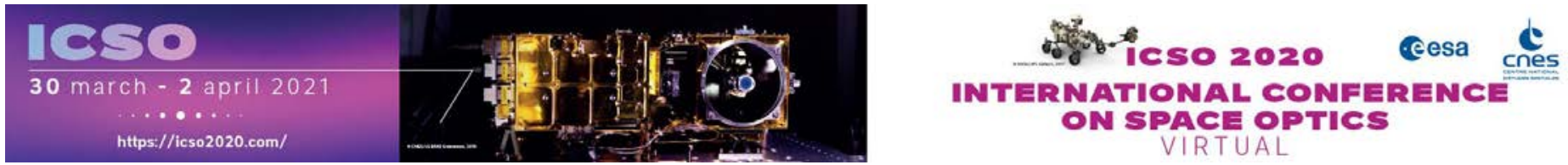

Development of full-field deflectometry for characterization of free-form mirrors for space applications

\section{Cesa isopmeatiant ecnes}




\title{
Development of full-field deflectometry for characterization of free-form mirrors for space applications
}

\author{
BOUWENS Arno ${ }^{(1)}$, BOUSSEMAERE Luc ${ }^{(1)}$, ANTOINE Philippe ${ }^{(1)}$, MOREAU Vincent $^{(2)}$, \\ BORGUET Benoit ${ }^{(2)}$, ARTS Mathijs ${ }^{(3)}$, TOMUTA Dana ${ }^{(3)}$, GEORGES Marc ${ }^{(4)}$, VANDENRIJT \\ Jean-François ${ }^{(4)}$.
}

(1) Lambda-X, Avenue Schuman 102, 141 Nivelles, Belgium

(2) AMOS, Rue des Chasseurs Ardennais 2, 4031 Angleur, Belgium

(3) ESTEC, Postbus 2992200 AG Noordwijk, The Netherlands

(4) Centre Spatial de Liège, Avenue du Pré-Aily 4031 Angleur - Belgium

\begin{abstract}
We demonstrate that full-field deflectometry is a viable alternative to interferometry for the characterization of free-form mirrors. Deflectometry does not require the use of a CGH. Instead of measuring the surface height map, the deflectometer measures the surface slopes in two orthogonal directions using the phase-shifting Schlieren method [1]. The surface height map is then reconstructed by integration of the slope maps. We present two instruments. The first one can be mounted in the lathe for in situ measurement. The second is adapted for the characterization of large concave mirrors.
\end{abstract}

Keywords: Freeform mirror, deflectometry, waviness, surface measurement

\section{INTRODUCTION}

The integration of freeform mirrors in optical systems is becoming more and more common as they allow optical designers to make systems more compact, more affordable and more performant. A crucial step during the manufacturing process of a freeform mirror is the characterization. Today, the standard method for the characterization of mirrors is interferometry. It can achieve sub-nanometer resolution, but only for almost flat or spherical surfaces. Therefore, freeform surface shapes require the integration of a null optic that compensates for the wavefront deformation introduced by the mirror under test. The null optic is typically a computer generated hologram (CGH) which needs to closely match the mirror being tested. Hence, each mirror requires its own (costly) CGH.

We demonstrate that full-field deflectometry is a viable alternative to interferometry for the characterization of free-form mirrors. Deflectometry does not require the use of a CGH. Instead of measuring the surface height map, the deflectometer measures the surface slopes in two orthogonal directions using the phase-shifting Schlieren method [1]. The surface height map is then reconstructed by integration.

We present two deflectometer instruments. The first instrument is designed to be mounted on the lathe, and allows in situ measurement of the mirror shape after machining. A second one allows measurement of large diameter concave mirrors. In contrast to the first one, we call is ex situ instrument. They are successively discussed in sections $2 \& 3$. 


\section{FULL-FIELD DEFLECTOMETER FOR IN SITU MEASUREMENT}

\subsection{Description}

A schematic of the experimental setup is displayed in Figure 1. The principle of the method is illustrated in Figure 2, with a configuration in transmission for simplicity, which is an unfolded version of the setup in reflection. The object under test (in purple) is imaged on the camera by a telecentric imaging system made of two lens assemblies and a pinhole that collects light rays that are parallel to the optical axis after refraction by the object under test. The illumination is placed at the focal place of the Schlieren objective. When there is no object, all the light rays collected by the pinhole, come from a single point, which is the intersection of the optical axis and the illumination plane. When the object is a wedge that deviates all the light rays in same way, the light rays originate from a single point but off-axis at the illumination plane (Figure 2b). The actual lighting means is a LED backlight and a spatial light modulator that generates a structured illumination pattern. As the illumination plane is the focal plane of the Schlieren lens, each light ray originates from a position whose distance to the optical axis is proportional to the incident angle $(\alpha)$ on the object under test. With structured illumination, original positions are converted into intensity. Figure 2c\&d illustrate the detected images with spherical lenses of low power and high power respectively. Rings are visible in the images since (i) refraction angles depend on the distances to the optical axis and (ii) the illumination pattern is periodic. In practice, illumination patterns, made of vertical stripes and horizontal stripes, are applied to measure deviations in the vertical and the horizontal planes successively. Angles are extracted from the intensity patterns by repeating the measurement for multiple phase-shifted illuminations and applying well-known phase-shifting algorithms. Finally, the phase maps are numerically unwrapped in order to calculate the deviation maps.

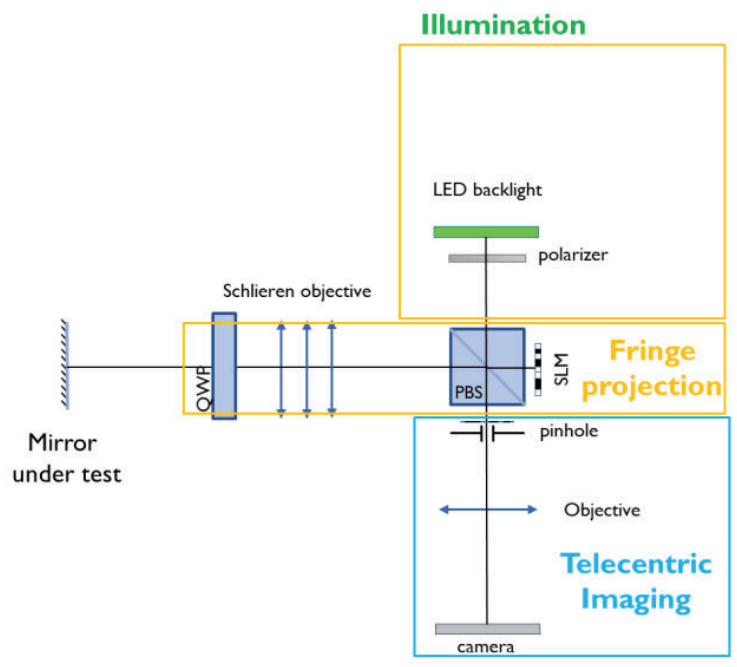

Figure 1:Schematic of the in situ experimental setup 


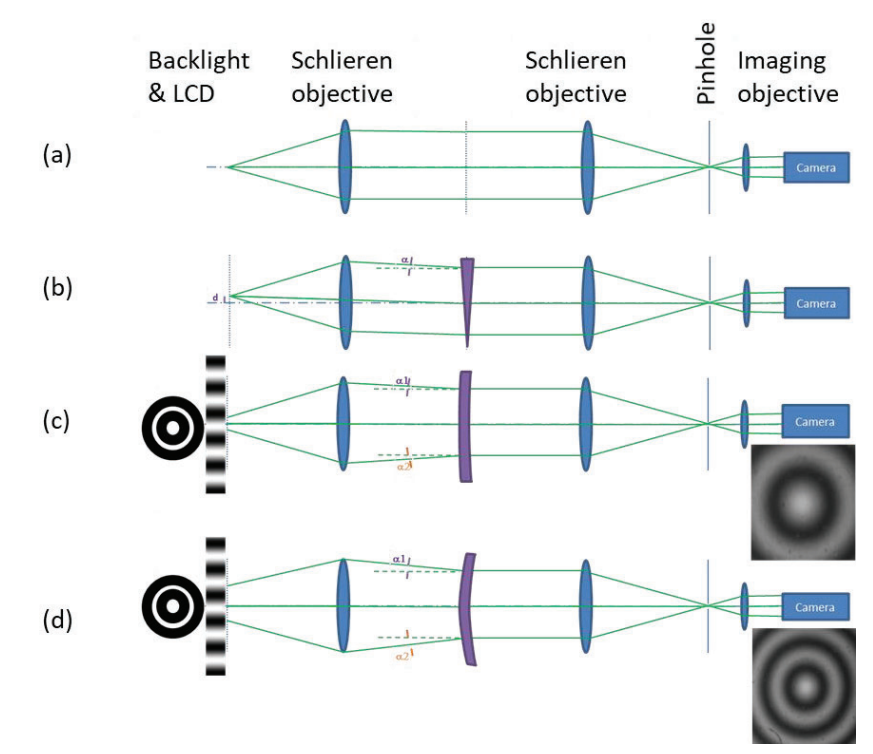

Figure 2: Principle of the Phase-Shifting Schlieren

In reflection, the Schlieren lens and the imaging lens are partially merged and a polarizing beam splitter is integrated to separate the illumination beam and the light rays reflected by the mirror. A quarter wave plate is integrated to rotate the polarization such that the beam reflected by the mirror is reflected towards the detector. Slope resolutions of $5 \mu$ rad and $25 \mu \mathrm{rad}$ were measured with coated and uncoated glass mirrors respectively. The lateral resolution is $85 \mu \mathrm{m}$ over a fieldof-view of $30 \mathrm{~mm}$. The angular acceptance is $\pm 1^{\circ}$. The field-of-view and the angular acceptance can be extended by subaperture stitching.

\subsection{Experimental results}

The so called "in-situ" experiment aims to demonstrate and characterize the deflectometer actual performances for condition where the system is directly integrated on the baseplate of a Single Point Diamond Turning lathe (SPDT).

The main benefits of in-situ metrology are:

- To provide accurate data for a quick correction of repeatable errors in a second iteration of SPDT.

. To provide a lower cost and universal alternative method (compared to CGH) for measuring highly aspherical or freeform mirrors.

The lathe is a Nanotech 350FG, an Ultra-Precision five axis CNC machining system for on-axis turning of aspheric and toroidal surfaces; slow-slide-servo machining (rotary ruling) of freeform surfaces; and raster fly-cutting of freeforms, linear diffractive, and prismatic optical structures.

The alignment of the instrument optical axis with the $\mathrm{C}$-axis of the lathe is required and quite straightforward. The working distance is not critical since the tolerances is around $20 \mathrm{~mm}$. It was set to $75 \mathrm{~mm}$ for the described experiment. Lateral adjustments (x-axis and y-axis) are performed with the help of rings projected by the instrument and a dedicated concave mirror stud. After this initial alignment, the metrology device can be removed and reinstalled in a perfectly repeatable position thanks to its line-point-plane base. 


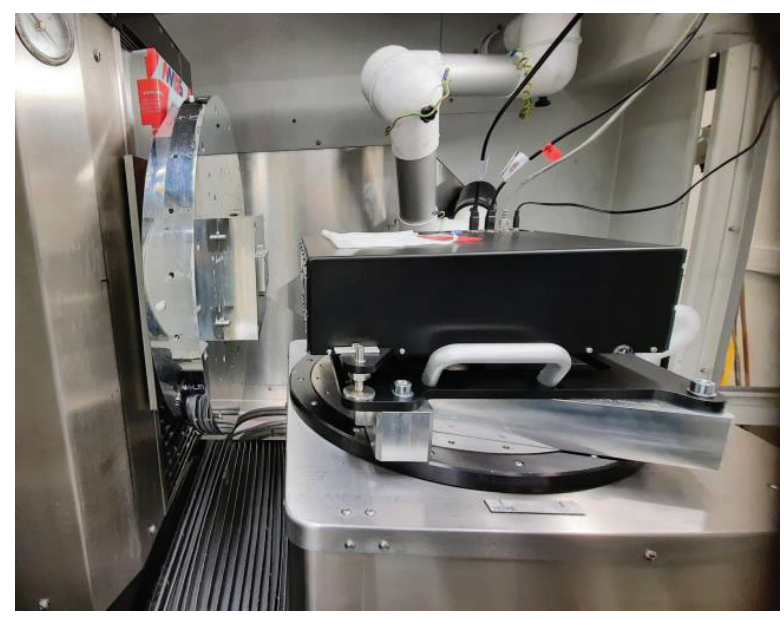

Figure 3 : Implementation of the deflectomer on the B-axis baseplate of the $350 \mathrm{FG}$ lathe

The phase measured by the deflectometer need to be converted to mirror slopes using an initial calibration. The phase values are multiplied by this sensitivity to obtain the corresponding mirror slopes. To achieve such a calibration, the instrument was tilted step-by-step by the lathe B-Axis in front of a flat mirror caliper (with surface error lower than $2 \mathrm{~nm}$ rms) and the deflectometer response was recorded. This calibration sequence also provides the offset error in each point (initial phase error due to in-field aberrations in the deflectometer), the linearity of the response, and the measurement noise for the defined acquisition parameters (Figure 4).

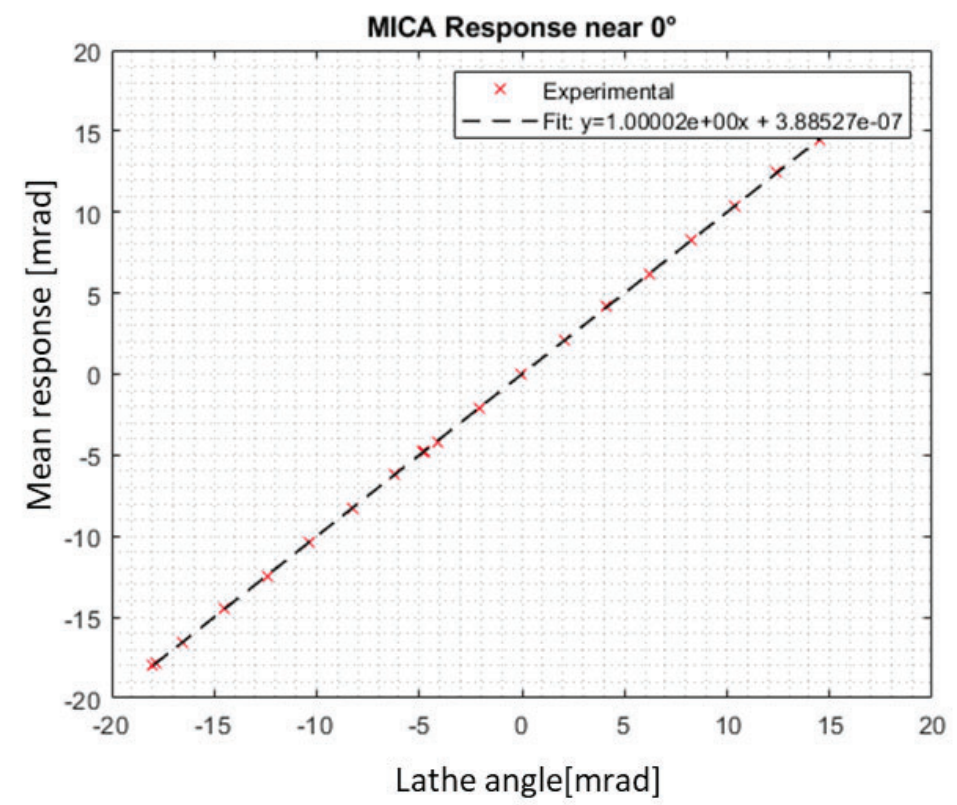

Figure 4: Averaged angle measured by the in situ instrument as a function of the orientation of the B-axis of the lathe 
Finally, an important objective of the in-situ test was to demonstrate the possible correction of repeatable manufacturing errors through combination of in-situ metrology and servo-tool control. The test was performed on a freeform mirror used for the correction of keystone and smile distortion in a high spectral resolution imaging spectrometer. The asdesigned mirror surface sag and slopes are presented in Figure 5.
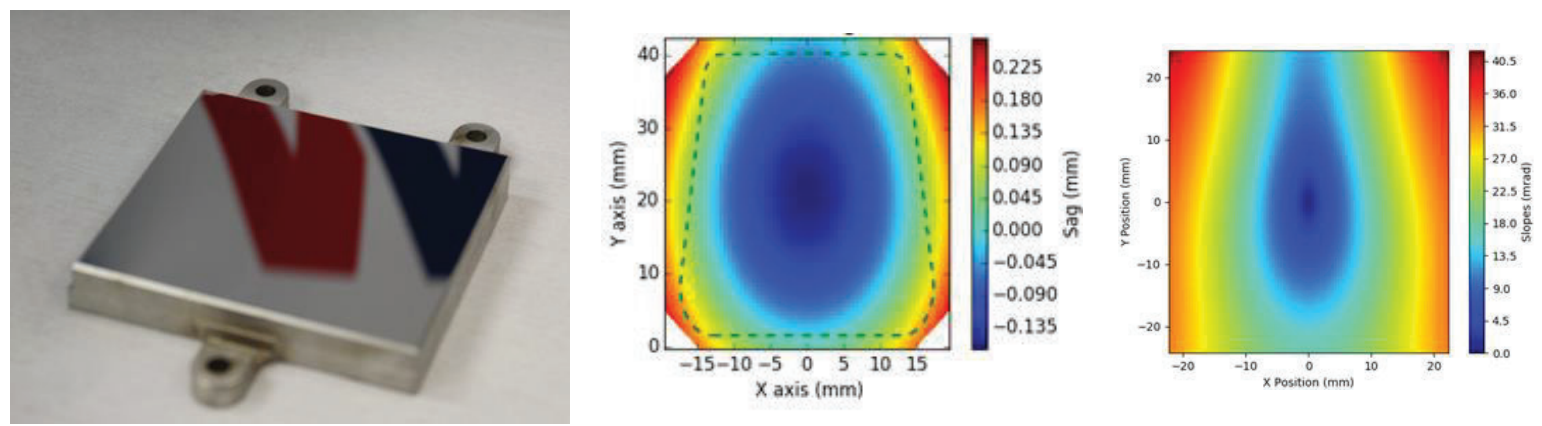

Figure 5 : Freeform mirror from CHIMA imaging spectrometer (left), Surface sag (Center) and slope map (right)

The following workflow has been implemented for this test (Figure 6).

The freeform mirror surface is first machined with Slide Servo Tool, using the nominal Zernike coefficient of the surface, as define in the optical design. A surface metrology is then performed in situ by deflectometry, the measured surface is projected in Zernike Function base and the coefficients are calculated. Measured Zernike coefficients are compared with the nominal (as designed) values and the error (difference) is computed for each coefficient. The initial coefficients are corrected by the measured errors and a new manufacturing run is operated. After completion of the second run, a new evaluation of the remaining error is performed with the deflectometer to estimate the convergence of the in-situ metrology approach.

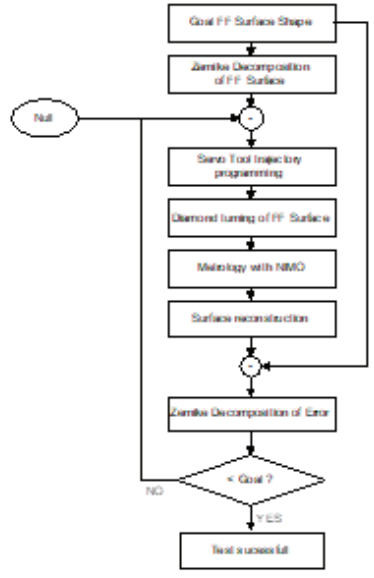

Figure 6: In situ correction test workflow

The achieved results are presented in Figure 7. The initial surface error was found to $102 \mathrm{~nm}$ rms. The corrective iteration resulted in a reduction of this error down to $81 \mathrm{~nm}$ rms. This initial result is particularly encouraging, especially when comparing the correction applied (bottom left) with the difference measured between the two iterations (bottom right) 

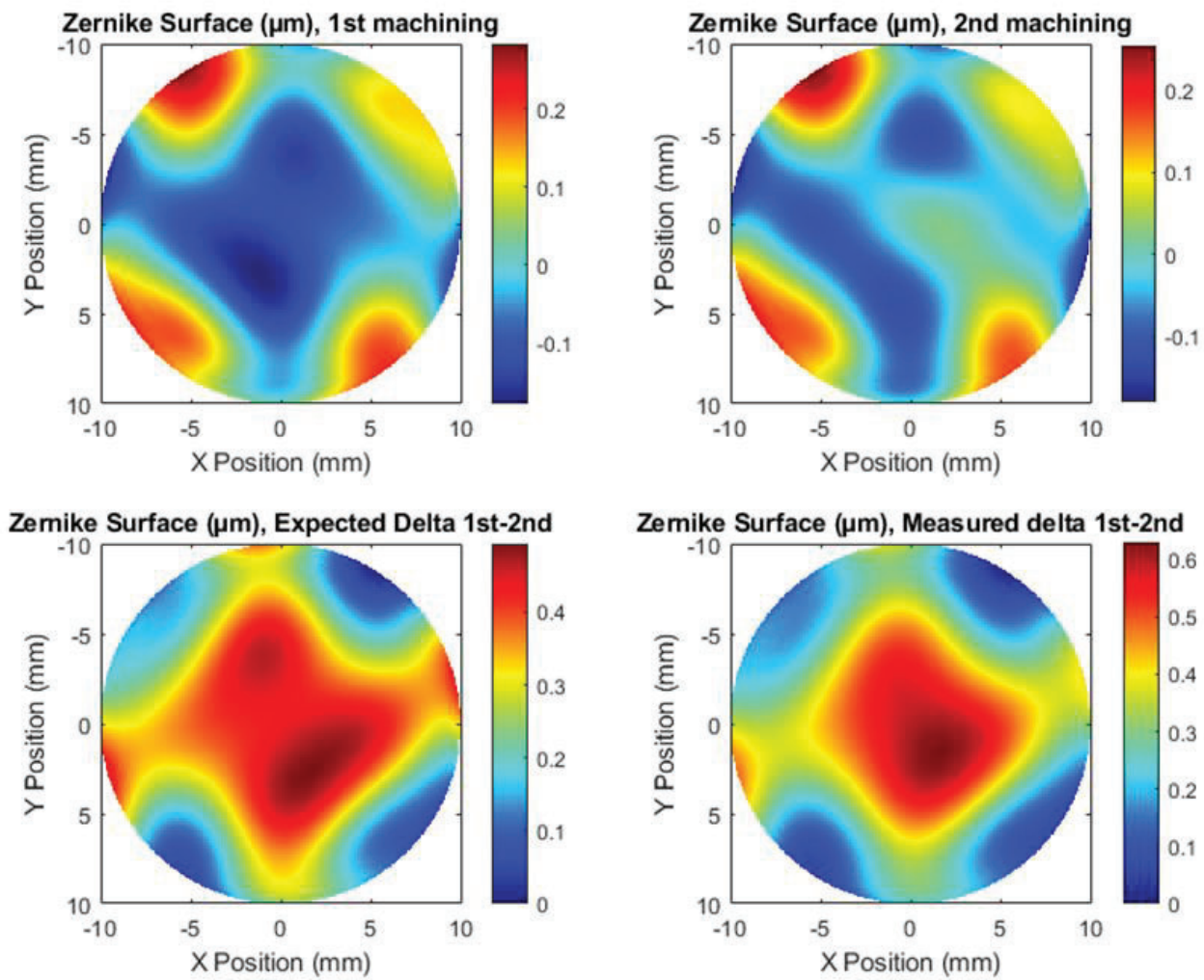

Figure 7: Results of the in-situ correction test. Top left : Initial measured SFE - Top right : Residual measured SFE after second machining - Bottom left : applied correction map - Bottom right : measured SFE difference between first and second machining.

\section{FULL-FIELD DEFLECTOMETER FOR CONCAVE MIRRORS}

\subsection{Description}

The instrument is based on phase-shifting deflectometry. The principle is illustrated in Figure 8. The mirror under test is illuminated by a LCD with a custom backlight. Reflected light is collected by an imaging system that is composed of a pinhole, a custom optical objective lens and a 1920x1200 CMOS camera. The pinhole is a critical component that defines the light rays after reflection on the mirror. In practice, to each pixel of the camera corresponds a direction of a light ray that is focused on the pinhole. Sinusoidal fringe patterns along the vertical and the horizontal directions are displayed successively on the LCD. The camera records images of the fringes for 4 different phases. For each pixel of the image, standard phase-shifting algorithm allows one to calculate the position on the LCD of the corresponding light ray. The reconstruction of the reflecting surface is not straightforward since there is no relation between the position on the LCD and the input direction on the mirror. Indeed, the position on the LCD depends not only on the orientation of the tested reflecting surface but also on the distance to the pinhole. In practice, the reflecting surface is reconstructed by an iterative algorithm that aims at reproducing the experimental result. 


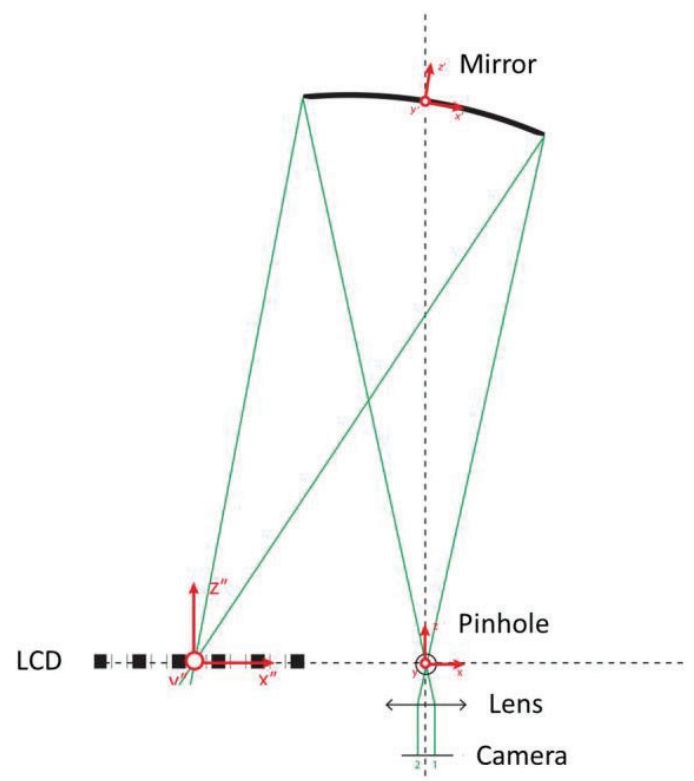

Figure 8: Schematic of the full-field deflectometer for concave mirrors

The principle of the instrument is similar to that of the in situ configuration (see section 2) when the Schlieren lens is removed. Moreover, it is preferred to move the LCD off-axis in order to avoid the beam splitter that would introduce aberrations given its size. With the proposed configuration, the system is aberration free since there is no optics, apart from the mirror under test, between the LCD and the pinhole. Therefore, the accuracy of the reconstruction depends critically on the relative 6-axis position of the LCD with respect to the pinhole: the 3D position (x"y"z"), the direction of the optical axis $\left(\theta_{x^{\prime \prime}}, \theta_{y^{\prime \prime}}\right)$ and the azimuth angle $\theta_{z^{\prime \prime}}$. The six coordinates were measured accurately during the calibration of the instrument. Similarly, the exact ray direction of each pixel of the camera was accurately determined during calibration as well.

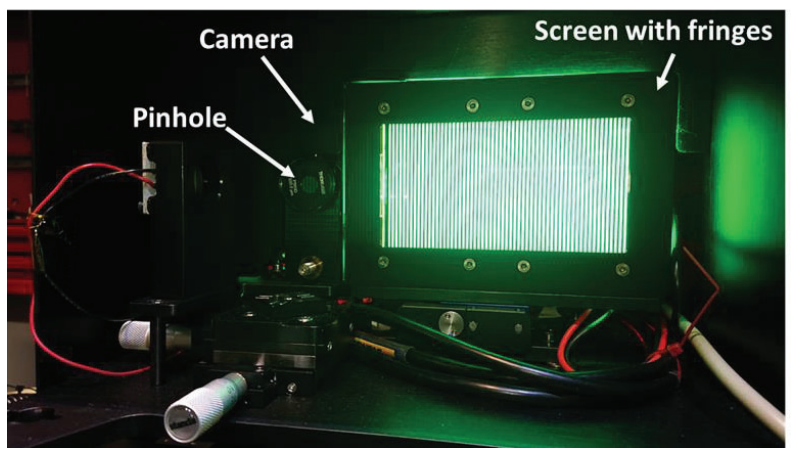

Figure 9: Picture of the instrument with horizontal fringes

An example of performance of the setup is depicted in Figure 10. We measured and reconstructed the surface of a spherical mirror (radius of curvature $=300 \mathrm{~mm}$ ). The deviation with respect to the best fitting sphere was then computed. The error equals $134 \mathrm{~nm}$ rms. Moreover, the slope accuracy is $20 \mu \mathrm{rad}$ rms while the slope resolution of the instrument is 
as low as $2 \mu \mathrm{rad}$. Therefore, it is worth noting that the performance could still be improved but it is currently limited by the calibration of the setup and not by its sensitivity.

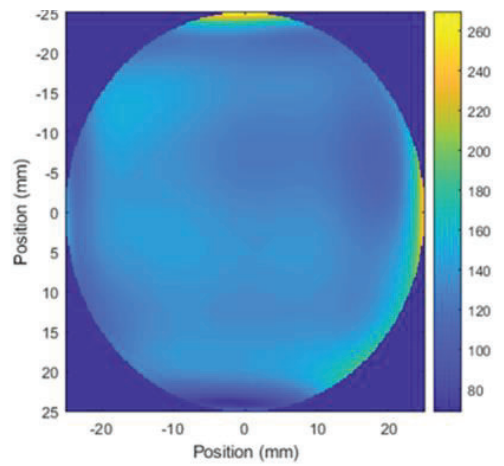

Figure 10: Surface deviation with respect to the best fitting sphere of a ROC $=300 \mathrm{~mm}$ spherical mirror

\subsection{Experimental results}

The investigated object is a spare space mirror from the Nomad spectrometer on board ESA's ExoMars trace gas orbiter [2]. The deformation of this mirror under thermal loading was previously measured by ESPI interferometry with high accuracy [3]. This previous measurement successfully matched a thermomechanical model with a difference of less than $30 \mathrm{~nm}$ RMS. The robustness of this ESPI measurement and, the possibility to reproduce it, comforted us to select this mirror as a reference test to assess the performances of the present instrument. The aim of our experiment is to measure the variations between an initial (room temperature) state and a hot state with the present instrument and compare the result with the ESPI measurement.

The Nomad mirror has a diameter of $84 \mathrm{~mm}$ and has an off-axis parabolic shape. The flat bottom surface is not parallel to the optical axis of the mirror, so it has been placed on a baseplate fixed to a tip-tilt stage. The baseplate is tightly clamped to maintain the mirror in position (see Figure 11b). The mirror is simply placed on its support in order to avoid mechanical stresses that are more complicated to simulate.

To heat the mirror and produce a deformation, a flexible polyimide thermo-foil heater from Minco has been glued on the mirror structure itself with an adhesive at the same position as the ESPI measurement described in [3]. The backside position of the heater (shown in Figure 11a) was originally selected in order to maximize the temperature gradient on the mirror, with the aims of producing deformations as large as possible.
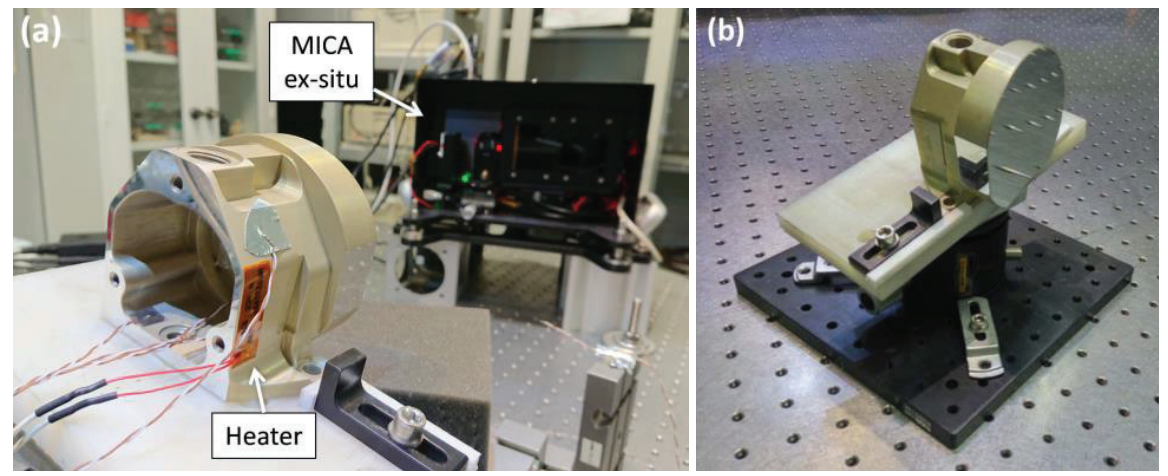

Figure 11: (a) Photo of the Nomad mirror on an insulator plate, thermal resistor, thermocouples, and MICA ex situ instrument in the back. (b) Mirror lying on the tip-tilt support maintained by stops on the insulator plate. 
After alignment of the instrument to minimize the number of fringes, 10 acquisitions at room temperature are performed to obtain the initial cold shape. After that, the instrument is set to perform automatic measurements every 10 minutes to monitor the shape variation, and the heater is powered at 2 Watts, the highest value that was set in the previous test to maximize the deformations [3]. The temperature of the mirror rises from room temperature, $\sim 21{ }^{\circ} \mathrm{C}$, to a stable hot temperature of $\sim 33^{\circ} \mathrm{C}$ in 3 hours (see Figure 12).

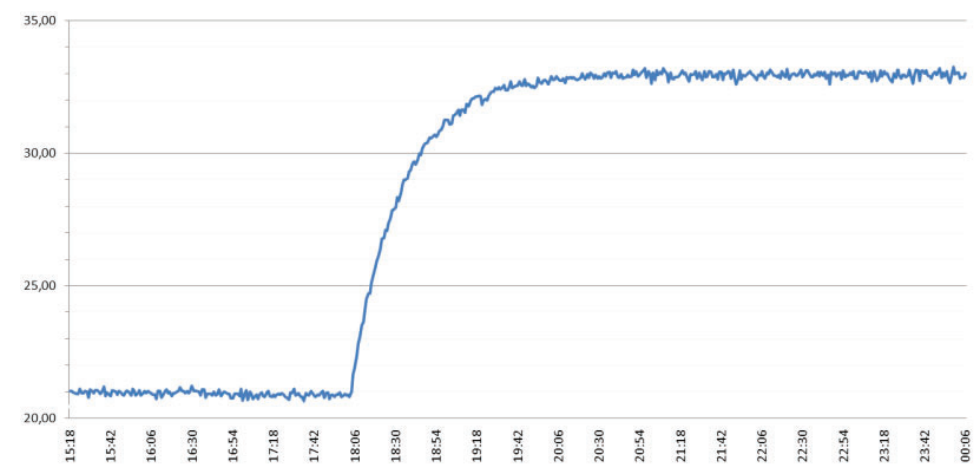

Figure 12: Temperature evolution of Nomad mirror during the thermal loading test.

Figure 13 shows the surface deformation observed by ESPI interferometry (left) and the new ex situ instrument (right). Figure 14 shows the difference between the two measurement after recentering the field-of-view relative to the ESPI measurement. A correct estimation of the deformation is computed. This result demonstrates the sensitivity of the ex situ instrument and its capability to reconstruct absolute surface. The discrepancy, smaller than $80 \mathrm{~nm}$, could be probably reduced by improving the calibration of the instrument. It is worth noting that both techniques, deflectometry and ESPI, are capable to follow the complete time evolution of the deformation while a classical interferometer requires realignment to compensate for the rigid-body shift of the surface which is the main effect due to the temperature increase.
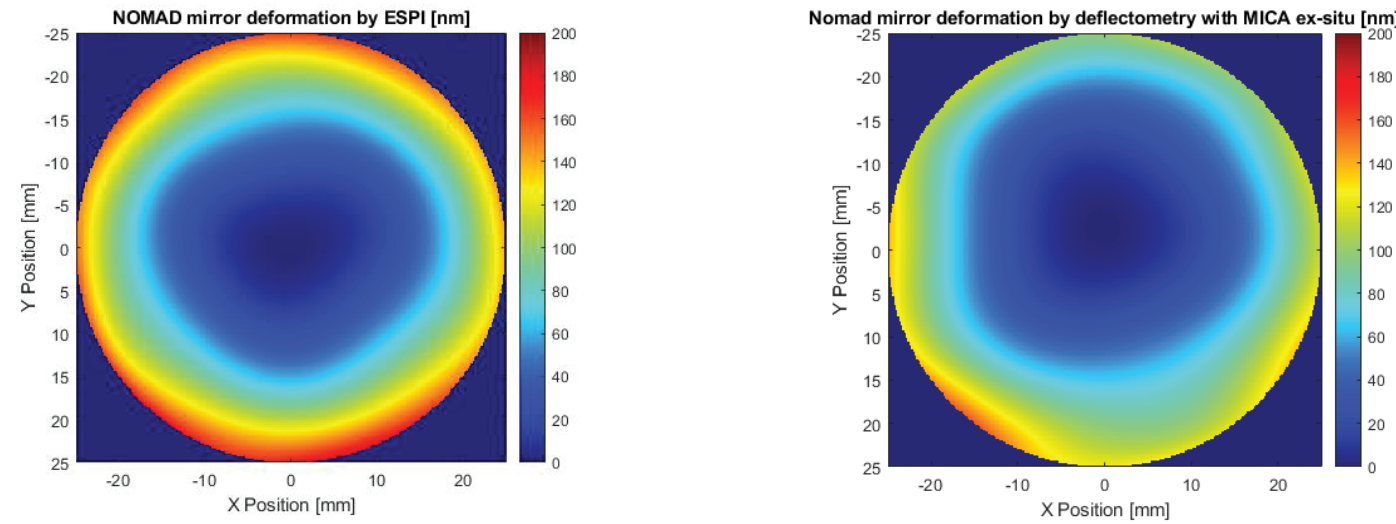

Figure 13: Comparison of the ESPI reference measurement and MICA ex situ measurement. 


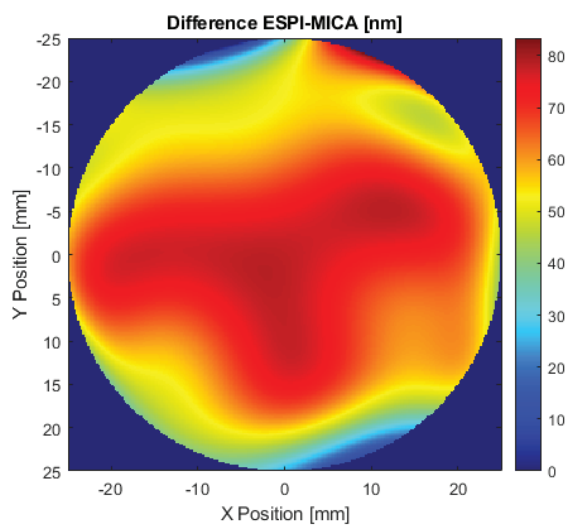

Figure 14: Difference between the MICA ex situ measurement and the ESPI reference measurement.

\section{ACKNOWLEGMENT}

The authors acknowledge the European Space Agency for financial support. The project was carried under the contract No. 4000123292/18/NL/GLC/fk.

The view expressed herein can in no way be taken to reflect the official opinion of the European Space Agency.

\section{REFERENCES}

[1] L. Joannes, F. Dubois and J.-C. Legos, "Phase-shifting schlieren: high-resolution quantitative schlieren that uses the phase-shifting technique principle," Appl. Opt. 42(25), 5046-5053 (2003).

[2] E. Neefs, A. C. Vandaele, R. Drummond, I. R. Thomas, S. Berkenbosch, R. Clairquin, S. Delanoye, B. Ristic, J. Maes, S. Bonnewijn, G. Pieck, E. Equeter, C. Depiesse, F. Daerden, E. Van Ransbeeck, D. Nevejans, J. Rodriguez-Gómez, J.-J. López-Moreno, R. Sanz, R. Morales, G. P. Candini, M. C. Pastor-Morales, B. Aparicio del Moral, J.-M. Jeronimo-Zafra, J. M. Gómez-López, G. Alonso-Rodrigo, I. Pérez-Grande, J. Cubas, A. M. Gomez-Sanjuan, F. Navarro-Medina, T. Thibert, M. R. Patel, G. Bellucci, L. De Vos, S. Lesschaeve, N. Van Vooren, W. Moelans, L. Aballea, S. Glorieux, A. Baeke, D. Kendall, J. De Neef, A. Soenen, P.-Y. Puech, J. Ward, J.-F. Jamoye, D. Diez, A. Vicario-Arroyo, and M. Jankowski, "NOMAD spectrometer on the ExoMars trace gas orbiter mission: part 1-design, manufacturing and testing of the infrared channels," Appl. Opt. 54(28), 8494-8520 (2015).

[3] F. Languy, J-F. Vandenrijt, Ph. Saint-Georges, S. Paquay, P. De Vincenzo and M. Georges, "Space mirror deformation: from thermo-mechanical measurements by speckle interferometry to optical comparison with multiphysics simulation," App. Opt. 57(24), 6982-6989 (2018). 